\section{Inquérito epidemiológico em escolares: determinantes e prevalência de fatores de risco cardiovascular}

\author{
Epidemiological survey in schoolchildren: \\ determinants and prevalence of \\ cardiovascular risk factors \\ Encuesta epidemiológica en escolares: \\ determinantes y prevalencia de factores de \\ riesgo cardiovascular
}

\section{Resumo}

Objetivou-se investigar a prevalência de dislipidemia, hiperglicemia e pressão arterial elevada em escolares, bem como, suas associações com fatores demográficos, socioeconômicos, biológicos e comportamentais. Estudo transversal com 1.139 escolares, de seis a 18 anos de idade, do Município de Amargosa, Bahia, Brasil. Foram analisadas variáveis demográficas, socioeconômicas, biológicas e comportamentais. Utilizou-se a razão de prevalência (RP) como medida de associação. As prevalências de dislipidemia, hiperglicemia e pressão arterial elevada foram, respectivamente, $62,1 \%, 6,6 \%$ e 27\%. A dislipidemia esteve associada com a localização geográfica da escola $(R P=1,52)$ e circunferência da cintura $(R P=1,20)$, e a hiperglicemia com a localização geográfica da escola $(R P=3,41)$ e adiposidade periférica $(R P=3,13)$. A pressão arterial elevada ficou associada com a faixa etária $(R P=2,34)$, razão da cintura pela estatura $(R P=1,62)$, maturação sexual $(R P=2,06)$ e atividade física $(R P=1,32)$. Programas de intervenção para escolares baseados em mudanças de hábitos de vida são necessários.

Estudantes; Doenças Cardiovasculares; Fatores de Risco;

Estudos Transversais
Teresa Maria Bianchini de Quadros 1 Alex Pinheiro Gordia 1

Luciana Rodrigues Silva 2 Diego Augusto Santos Silva 3 Jorge Mota 4

\footnotetext{
1 Universidade Federal do Recôncavo da Bahia, Amargosa, Brasil. 2 Universidade Federal da Bahia, Salvador, Brasil. 3 Universidade Federal de Santa Catarina, Florianópolis, Brasil. 4 Universidade do Porto, Porto, Portugal.
}

\author{
Correspondência \\ A. P. Gordia \\ Universidade Federal do \\ Recôncavo da Bahia. \\ Rua A 55, 1o andar, Amargosa, \\ BA 45300-000, Brasil. \\ alexgordia@gmail.com
}




\section{Introdução}

As doenças que afetam a população são produtos da organização social e a ocorrência destas enfermidades reflete em primeira instância os determinantes socioepidemiológicos da sociedade 1,2 . Muito se reportou que as doenças e agravos não transmissíveis à saúde, como as doenças cardiovasculares, dislipidemias, hipertensão arterial e diabetes tipo 2 são ocasionadas pelo estilo de vida individual da população. Porém, esses e outros agravos à saúde devem ser entendidos como produtos de um contexto ecológico que sofre influência das relações econômicas, sociais e políticas que, por sua vez, irão afetar os hábitos individuais dos sujeitos 3,4. Dessa forma, entender essas e outras doenças, compreende debater as iniquidades sociais em saúde que vão além dos aspectos individuais.

A dislipidemia, a hiperglicemia e a pressão arterial elevada estão entre os principais fatores de risco independentes para doenças cardiovasculares, sendo responsáveis por quase um quarto das causas de morte em todo o mundo 5 . No Brasil, um número significativo de internações hospitalares e custos estimados em mais de 750 milhões de dólares por ano são atribuídos às doenças cardiovasculares 6 . Nesse sentido, o enfrentamento aos principais fatores de risco associados a essas patologias tem ganhado espaço na agenda nacional de saúde pública 7 .

Prevalências preocupantes de dislipidemia, hiperglicemia e pressão arterial elevada também têm sido observadas na população pediátrica 8,9,10,11,12,13,14,15. A presença desses fatores de risco na infância e adolescência aumenta significativamente a chance para o surgimento de doenças cardiovasculares na vida adulta 16 , representando uma elevada carga de morbidade e mortalidade precoce na população ${ }^{5}$.

Fatores demográficos e socioeconômicos podem ser entendidos como parte das relações econômicas, sociais e políticas que exercem influência sobre as doenças e os agravos à saúde 3,4 . Pesquisas já demonstraram que tais fatores foram determinantes para o desenvolvimento de dislipidemia, hiperglicemia e pressão arterial elevada na população pediátrica 8,9,10,11,12. O que agrava essa inter-relação é que esses determinantes exercem impactos também nos aspectos biológicos e comportamentais que, por sua vez, afetam o desenvolvimento de doenças e agravos à saúde durante a infância e adolescência 8,9,10,11,12,14. No entanto, no Brasil, em especial na Região Nordeste, é limitado o corpo de evidências sobre a prevalência e fatores associados a esses desfechos na população pediátrica, sobretudo em municípios de pequeno porte. A identificação de regiões e comunidades em que os fatores de risco à saúde são prevalentes e o entendimento de variáveis associadas a esses desfechos representam etapas fundamentais para o direcionamento de estratégias mais eficazes de vigilância das doenças crônicas não transmissíveis em idades precoces.

Com base nesse contexto, o objetivo do presente estudo foi investigar a prevalência de dislipidemia, hiperglicemia e pressão arterial elevada em escolares de um município de pequeno porte do Nordeste do Brasil, bem como, suas associações com fatores demográficos, socioeconômicos, biológicos e comportamentais.

\section{Métodos}

\section{População e amostra}

O presente trabalho faz parte de um inquérito epidemiológico de base escolar desenvolvido no Município de Amargosa, Bahia, Região Nordeste do Brasil, considerado de pequeno porte, com população estimada em 34.845 habitantes para o ano de 2012 e índice de desenvolvimento humano (IDH) de 0,625. O IDH do município está abaixo da média brasileira $(0,744)$, muito próximo ao de outros municípios das regiões Norte e Nordeste do Brasil e de países menos desenvolvidos como Guatemala $(0,628)$ e Timor-Leste $(0,620)$. Essas características demonstram a vulnerabilidade econômica e social do município e a relevância da realização de inquéritos epidemiológicos que possibilitem o planejamento de políticas públicas mais eficazes de promoção da saúde.

A população do estudo foi composta por escolares de ambos os sexos, com idades entre seis a 18 anos, alunos do 1o ao 9o ano do Ensino Fundamental e do 1o ao 3 o ano do Ensino Médio das redes pública e particular do município. De acordo com dados da Secretaria de Educação do Município, no ano de 2011 estavam matriculados nesse segmento educacional 7.708 estudantes, distribuídos em 42 escolas, sendo 40 públicas, 13 urbanas $(n=5.207)$ e 27 rurais $(n=1.853)$, e duas particulares $(\mathrm{n}=648)$. O município estudado tem uma extensão territorial de $435,932 \mathrm{~km}^{2}$. Por esse motivo, a Secretaria Municipal de Educação dividiu o território em seis núcleos educacionais (um urbano e cinco rurais) que englobam toda a área do município. Dentre os núcleos da área rural, o menor tem duas escolas e o maior era composto por sete escolas no ano de 2011. Na área urbana, todas as escolas estavam alocadas no mesmo núcleo.

Para o cálculo do tamanho da amostra representativa da população, utilizou-se a metodologia recomendada por Luiz \& Magnanini 17, 
baseando-se em prevalência estimada em 50\%, nível de 95\% de confiança e precisão de três pontos percentuais, obtendo-se o tamanho amostral de 971 escolares. Empregou-se acréscimo de 20\% para os possíveis casos de perdas ou recusas, totalizando 1.165 escolares. Essa amostra também tem poder para detectar como significantes razões de prevalências (RP) iguais ou superiores a $1,30 \mathrm{e} / \mathrm{ou}$ iguais ou inferiores a 0,70 , com a prevalência do desfecho variando de $32,5 \%$ a $97,5 \%$ nos expostos e de $25 \%$ a $75 \%$ nos não expostos.

O procedimento de seleção amostral foi realizado em dois estágios, sendo que a "escola" foi a unidade amostral primária e o "escolar" foi a secundária. No primeiro estágio, utilizou-se o procedimento amostral por conglomerado de escolas com estratificação proporcional por tipo de escola ("públicas urbanas", "públicas rurais" e "particulares") e por núcleo educacional para as escolas da área rural (visando a garantir a distribuição geográfica da amostra da área rural). Foram sorteadas cinco escolas públicas urbanas, cinco públicas rurais (uma de cada núcleo de estudo) e uma particular, com a estimativa de tamanho amostral para cada estrato sendo proporcional ao observado na população de estudo (públicas urbanas: $\mathrm{n}=787$; públicas rurais: $\mathrm{n}=$ 280; particular: $n=98$ ). No segundo estágio, os escolares foram selecionados por sorteio simples, considerando o número de indivíduos necessários em cada escola para compor a amostra de forma proporcional ao tamanho (número de alunos matriculados) da escola. A coleta de dados foi realizada de agosto de 2011 a maio de 2012. Todas as avaliações foram feitas na própria escola durante o período matutino.

\section{Instrumentos e procedimentos}

\section{- Variáveis dependentes}

Perfil lipídico e glicêmico: amostras de sangue venoso $(10 \mathrm{~mL})$ foram coletadas nas escolas, pela manhã, após jejum de 12 horas. As análises da glicemia, dos triglicerídeos e do colesterol total e frações foram determinadas usando-se um analisador bioquímico automático da marca Biosystems, modelo A15 (Biosystems, Curitiba, Brasil). A glicemia foi verificada por meio do método enzimático com base na análise do plasma fluoretado. A verificação do colesterol total, triglicerídeos, HDL-C e LDL-C foi realizada com base na análise do soro, sendo que para o colesterol total, HDL-C e triglicerídeos utilizou-se o método enzimático, e para o LDL-C recorreu-se à fórmula de Friedewald et al. 18 .

O diagnóstico de dislipidemia foi definido como a presença de uma ou mais das seguin- tes alterações lipídicas: colesterol total elevado ( $\geq 170 \mathrm{mg} / \mathrm{dL}$ ), HDL-C baixo (<45mg/dL), LDL-C elevado $(\geq 130 \mathrm{mg} / \mathrm{dL}$ ) e concentração elevada de triglicerídeos ( $\geq 130 \mathrm{mg} / \mathrm{dL}$ ). Os pontos de corte utilizados para cada uma das alterações lipídicas basearam-se na I Diretriz de Prevenção da Aterosclerose na Infância e Adolescência 19. A presença de hiperglicemia foi definida pela concentração de glicemia em jejum $\geq 100 \mathrm{mg} / \mathrm{dL} 20$.

Pressão arterial sistólica e diastólica: a medida dos níveis pressóricos foi realizada utilizando-se monitor digital e automático Omron, modelo HEM742 INT (Omron Healthcare, Inc., Bannockburn, Estados Unidos), e manguitos de tamanho apropriado à circunferência do braço das crianças e adolescentes avaliados. A medida foi realizada no braço direito à altura do coração após o estudante permanecer cinco minutos em repouso. A pressão arterial elevada foi classificada como pressão arterial sistólica ou diastólica $\geq$ percentil 9521 .

\section{- Variáveis independentes}

Variáveis demográficas e socioeconômicas: a idade (crianças: 6-9 anos e adolescentes: 10-18 anos), o sexo (feminino e masculino), a cor da pele (branca e outras), a localização geográfica da escola (urbana e rural), o tipo de escola (pública e particular), o número de irmãos (nenhum, 1-2 e $\geq 3$ ), o número de indivíduos por residência $(\leq 3,4-5$ e $\geq 6)$, a escolaridade materna em anos $(<4,4-8,>8)$, a renda familiar mensal em salários mínimos $(<1$ e $\geq 1)$ e as classes econômicas foram obtidas pelo autorrelato dos escolares e dos pais por meio de entrevista. Para avaliar as classes econômicas, utilizou-se o Critério de Classificação Econômica Brasil 22. Esse critério determina as classes econômicas com base na estimativa do poder de compra das pessoas e famílias investigadas. As classes econômicas foram agrupadas em "AB", "C" e "DE".

Variáveis biológicas: mensurou-se a massa corporal por meio de uma balança digital Plenna (Plenna, São Paulo, Brasil), com capacidade para $150 \mathrm{~kg}$ e resolução de $100 \mathrm{~g}$, e a estatura por meio de um estadiômetro portátil, fixado à parede, da marca Seca (Seca, Cotia, Brasil), graduado de 0 a $220 \mathrm{~cm}$, com escala de precisão de $0,1 \mathrm{~cm}$. As duas variáveis foram mensuradas de acordo com procedimentos e técnicas padronizadas 23 e utilizadas para o cálculo do índice de massa corporal (IMC). Para a classificação do IMC foram utilizados os pontos de corte propostos por Cole et al. ${ }^{24}$. Os escolares foram classificados como "peso normal" ou "excesso de peso" (sobrepeso ou obesidade). A circunferência da cintura (CC) foi mensurada por meio de uma 
fita antropométrica inelástica com resolução de $0,1 \mathrm{~cm}$, com base nos procedimentos descritos pela Organização Mundial da Saúde (OMS) 25 e classificada como normal ou elevada 26 . As medidas da estatura e da CC foram utilizadas para o cálculo da razão da cintura pela estatura (RCEst). Esse indicador foi utilizado porque tem a vantagem adicional em relação a CC de considerar as mudanças na estatura que ocorrem em virtude do crescimento físico. Foram considerados com a RCEst elevada os escolares que apresentaram valor $\geq 0,5$. Para a verificação da adiposidade central e periférica foram mensuradas as espessuras das dobras cutâneas (DC) subescapular (SE) e triciptal (TR), respectivamente. Para tanto, foi utilizado um plicômetro científico da marca Cescorf (Porto Alegre, Brasil) com resolução de 0,1 mm, e a mensuração seguiu procedimentos e técnicas padronizadas 27 . As medidas foram obtidas no lado direito dos estudantes e repetidas três vezes sucessivas em cada local. Utilizou-se como valor a média das três medidas. Considerou-se como excesso de adiposidade central e periférica valores iguais e/ou superiores ao percentil $90 \mathrm{da}$ curva de referência do National Center for Health Statistics dos Estados Unidos 28.

Para avaliação da maturação sexual foi utilizado o método proposto por Tanner ${ }^{29}$. O teste foi aplicado por intermédio da autoavaliação do desenvolvimento da pilosidade pubiana 30 . Os escolares foram classificados em pré-púberes (estágio I), púberes (estágios II, III e IV) e póspúberes (estágio V).

Variáveis comportamentais: a prática de atividade física foi avaliada usando-se o pedômetro da marca Yamax, modelo Digi-walker SW-200 (Yamasa Tokei Keiki Co., Tóquio, Japão), durante uma semana, incluindo o fim de semana. Para a classificação da atividade física foram adotados os pontos de corte propostos por Duncan et al. 31 .

O comportamento sedentário dos estudantes foi investigado pela pergunta: "em média quantas horas você assiste à televisão por dia?”, e classificado com base no tempo assistindo à TV igual ou superior a duas horas por dia 32 .

\section{Qualidade das informações coletadas}

Os questionários foram aplicados por meio de entrevista realizada por um grupo de dez avaliadores previamente treinados em estudo piloto, todos discentes e docentes do curso de Licenciatura em Educação Física da Universidade Federal do Recôncavo da Bahia. As amostras de sangue foram coletadas por técnicos em enfermagem funcionários da Prefeitura Municipal de Amargosa, e transportadas sob refrigeração para análise no laboratório de análises clínicas do município.
As avaliações antropométricas foram realizadas no período da manhã (após a coleta sanguínea) por dois avaliadores, um do sexo feminino e um do sexo masculino. Com o intuito de evitar constrangimentos, as medidas antropométricas e as orientações para autoavaliação da maturação sexual foram realizadas por avaliadores do mesmo sexo dos escolares. Visando a garantir a confiabilidade das medidas antropométricas investigadas, foi calculado o erro técnico de medida intra-avaliador e interavaliador, considerando a metodologia recomenda por Norton \& Olds 33. Ambos os avaliadores apresentaram erro técnico de medida intra-avaliador e interavaliador para as medidas de DC inferior a $5 \%$ e $7,5 \%$, respectivamente, e para as demais medidas, inferior a $1 \%$ e $1,5 \%$, respectivamente, percentuais de erro considerados aceitáveis 33 .

\section{Análise estatística}

Foram calculadas as frequências absolutas e relativas, intervalos de 95\% de confiança (IC95\%), média e desvio padrão (DP). Para comparar a prevalência das variáveis dependentes por categorias das variáveis independentes utilizou-se o teste de Wald para heterogeneidade e tendência linear. Tanto para a análise bruta quanto para a ajustada, a RP foi utilizada como medida de associação, estimada por meio da regressão de Poisson com variância robusta, e o teste de Wald foi adotado para testar a significância estatística. Para a modelagem estatística da análise ajustada adotou-se o método de entrada backward stepwise e ordem de ingresso das variáveis conforme modelo conceitual hierarquizado com três níveis: (1) variáveis demográficas e socioeconômicas - faixa etária, sexo, cor da pele, número de irmãos, número de pessoas que residem na casa, escolaridade da mãe, localização geográfica da escola, tipo de escola, renda familiar mensal, classe econômica; (2) variáveis biológicas - IMC CC, RCEst, DCSE, DCTR, maturação sexual; e (3) variáveis comportamentais - prática de atividade física e tempo assistindo à TV. Permaneceram no modelo de regressão as variáveis que apresenta$\operatorname{ram} \mathrm{p} \leq 0,20$ para controle de fatores de confusão. Foram considerados fatores associados ao desfecho as variáveis com valor de $\mathrm{p} \leq 0,05$. Os dados foram analisados no programa IBM SPSS, versão 20.0 (IBM Corp., Armonk, Estados Unidos). Para o cálculo do poder amostral do estudo realizado a posteriori utilizou o programa Epi Info, versão 6.04 (Centers for Disease Control and Prevention, Atlanta, Estados Unidos). 


\section{Critérios éticos}

O protocolo do presente trabalho foi aprovado pelo Comitê de Ética em Pesquisa da Faculdade Maria Milza (processo no 126/2011). Foram incluídos no estudo apenas os escolares que aceitaram participar voluntariamente e tiveram autorização prévia dos pais ou responsável legal, mediante assinatura de termo de consentimento livre e esclarecido.

\section{Resultados}

Foram avaliados 1.139 estudantes, sendo 769 de escolas públicas urbanas, 275 de escolas públicas rurais e 95 da escola particular, havendo $2,2 \%$ de perdas por recusa ou ausência no dia da coleta de dados. A média de idades foi de 11,5 anos (DP = 3,3). A Tabela 1 apresenta a descrição das variáveis investigadas. A amostra foi composta por um número superior de escolares do sexo feminino, adolescentes, com cor da pele não branca, estudantes da área urbana, de escolas públicas e com renda familiar mensal inferior a um salário mínimo. Mais de 60\% da amostra relataram escolaridade materna inferior a oito anos de estudos e aproximadamente $40 \%$ pertenciam às classes econômicas D e E. O comportamento sedentário, a prática insuficiente de atividade física e a dislipidemia foram, nesta ordem, os fatores de risco cardiovascular mais prevalentes. As prevalências de colesterol total elevado, HDL-C baixo, LDL-C elevado e triglicerídeos elevado foram de $23,1 \%$ (IC95\%: 20,7-25,7), 41,5\% (IC95\%: 38,6-44,4), 4,5\% (IC95\%: 3,4-5,8) e 12,4\% (IC95\%: 10,6$14,4)$, respectivamente. A simultaneidade de dislipidemia, hiperglicemia e pressão arterial elevada foi observada em 1,8\% (IC95\%: 1,2-2,8) dos escolares investigados.

Na análise bruta, a localização geográfica da escola, o IMC, a RCEst, a adiposidade central e a periférica estiveram associados à dislipidemia, à hiperglicemia e à pressão arterial elevada, sendo que jovens que estudavam em escolas urbanas, com excesso de peso, com RCEst elevada e com adiposidade central e periférica elevadas foram mais prováveis para apresentar os desfechos (RP variando de 1,26 a 3,48). A faixa etária, a CC e a maturação sexual estiveram associadas à dislipidemia e à pressão arterial elevada, com maior probabilidade para presença dos desfechos entre adolescentes, indivíduos com CC elevada, púberes e pós-púberes (RP variando de 1,15 a 3,75). Além disso, a atividade física esteve associada à pressão arterial elevada, sendo que jovens insuficientemente ativos foram mais prováveis para apresentar o desfecho $(\mathrm{RP}=1,45)$

(Tabelas 2, 3 e 4).

$\mathrm{Na}$ análise ajustada, estudantes de escolas urbanas (RP = 1,52; IC95\%: 1,31-1,76) e aqueles com CC elevada (RP = 1,20; IC95\%: 1,10-1,32) apresentaram maior probabilidade para ter dislipidemia (Tabela 2). Para a hiperglicemia, após ajuste por fatores de confusão, tanto estudantes de escolas urbanas quanto aqueles com adiposidade periférica elevada apresentaram probabilidade superior a três vezes para ter hiperglicemia $(\mathrm{RP}=3,41$; IC95\%: 1,48-7,87 e RP = 3,13; IC95\%: 1,41-6,97, respectivamente) (Tabela 3). Quanto à análise ajustada para a pressão arterial elevada, os subgrupos de escolares com maior probabilidade para apresentar este desfecho foram compostos por adolescentes $(\mathrm{RP}=2,34$; IC95\%: 1,50-3,64), escolares com RCEst elevada ( $\mathrm{RP}=1,62$; IC95\%: 1,26-2,08), púberes (RP = 1,77; IC95\%: 1,16-2,70), pós-púberes ( $\mathrm{RP}=2,06$; IC95\%: 1,21-3,50) e insuficientemente ativos (RP = 1,32; IC95\%: 1,02-1,71) (Tabela 4).

O cálculo do poder amostral realizado $a$ posteriori para cada um dos desfechos analisado indicou que para a prevalência de dislipidemia encontrada no presente estudo, a amostra foi capaz de detectar como significantes razões de prevalências iguais ou superiores a 1,22 e/ou iguais ou inferiores a 0,82 , respectivamente, para fatores de risco e proteção, com a prevalência da exposição variando de $10 \%$ a $45 \%$. Para a prevalência de hiperglicemia, a amostra foi capaz de detectar como significantes razões de prevalências iguais ou superiores a 2,75 e/ou iguais ou inferiores a 0,36 , respectivamente, para risco e proteção, com a prevalência da exposição variando de $5 \%$ a $45 \%$. Para a prevalência de pressão arterial elevada, a amostra foi capaz de detectar como significantes razões de prevalências iguais ou superiores a 1,65 e/ou iguais ou inferiores a 0,60 , respectivamente, para risco e proteção, com a prevalência da exposição variando de $5 \%$ a $45 \%$.

\section{Discussão}

A dislipidemia, a hiperglicemia e a pressão arterial elevada foram fatores de risco presentes entre crianças e adolescentes residentes em um município de pequeno porte situado no interior do Estado da Bahia. Indicadores antropométricos de obesidade estiveram associados aos três fatores de risco investigados, ao passo que a localização geográfica da escola esteve associada à dislipidemia e à hiperglicemia. Em adição, a faixa etária, a maturação sexual e a atividade física registraram associação com a pressão arterial elevada. Esses resultados podem ser úteis para auxiliar no 
Descrição das variáveis demográficas, socioeconômicas, maturação sexual e prevalência de fatores de risco cardiovascular dos escolares, com valores expressos em frequência absoluta, relativa e intervalo de 95\% de confiança (IC95\%). Amargosa, Bahia, Brasil, 2011-2012.

\begin{tabular}{|c|c|c|}
\hline Variáveis & $\mathbf{n}$ & $\%(I C 95 \%)$ \\
\hline \multicolumn{3}{|l|}{ Sexo } \\
\hline Masculino & 506 & $44,4(41,6-47,3)$ \\
\hline Feminino & 633 & $55,6(52,7-58,4)$ \\
\hline \multicolumn{3}{|l|}{ Faixa etária (anos) } \\
\hline Crianças (6-9) & 363 & $31,9(29,2-34,6)$ \\
\hline Adolescentes (10-18) & 776 & $68,1(65,4-70,8)$ \\
\hline \multicolumn{3}{|l|}{ Cor da pele } \\
\hline Branca & 222 & $19,6(17,4-22,0)$ \\
\hline Outras & 911 & $80,4(78,0-82,6)$ \\
\hline \multicolumn{3}{|l|}{ Localização geográfica da escola } \\
\hline Área urbana & 864 & $75,9(73,3-78,3)$ \\
\hline Área rural & 275 & $24,1(21,7-26,7)$ \\
\hline \multicolumn{3}{|l|}{ Tipo de escola } \\
\hline Pública & 1.044 & $91,7(90,0-93,0)$ \\
\hline Particular & 95 & $8,3(6,8-9,7)$ \\
\hline \multicolumn{3}{|l|}{ Número de irmãos } \\
\hline Nenhum & 112 & $10,0(8,3-11,9)$ \\
\hline $1-2$ & 567 & $50,4(47,5-53,4)$ \\
\hline$\geq 3$ & 445 & $39,6(36,8-42,5)$ \\
\hline \multicolumn{3}{|l|}{ Número de pessoas por residência } \\
\hline$\leq 3$ & 239 & $21,1(18,9-23,6)$ \\
\hline $4-5$ & 574 & $50,8(47,9-53,7)$ \\
\hline$\geq 6$ & 317 & $28,1(25,5-30,7)$ \\
\hline \multicolumn{3}{|l|}{ Escolaridade materna (anos) } \\
\hline$<4$ & 264 & $24,5(22,1-27,2)$ \\
\hline $4-8$ & 389 & $36,2(33,3-39,1)$ \\
\hline$>8$ & 423 & $39,3(36,4-42,3)$ \\
\hline \multicolumn{3}{|l|}{ Renda familiar mensal (salário mínimo) * } \\
\hline$<1$ & 614 & $57,2(54,2-60,2)$ \\
\hline$\geq 1$ & 459 & $42,8(39,8-45,8)$ \\
\hline \multicolumn{3}{|l|}{ Classe econômica } \\
\hline$A B$ & 141 & $12,4(10,6-14,4)$ \\
\hline C & 550 & $48,4(45,5-51,3)$ \\
\hline DE & 446 & $39,2(36,4-42,1)$ \\
\hline \multicolumn{3}{|l|}{ Maturação sexual } \\
\hline Pré-púbere & 493 & $43,4(40,5-46,3)$ \\
\hline Púbere & 568 & $50,0(47,1-52,9)$ \\
\hline Pós-púbere & 75 & $6,6(5,3-8,2)$ \\
\hline Comportamento sedentário & 947 & $83,1(80,9-85,2)$ \\
\hline Prática insuficiente de atividade física & 696 & $64,3(61,4-67,1)$ \\
\hline Excesso de peso & 181 & $15,9(13,9-18,1)$ \\
\hline Circunferência da cintura elevada & 529 & $46,4(43,6-49,3)$ \\
\hline Razão cintura/estatura elevada & 224 & $19,7(17,5-22,1)$ \\
\hline Excesso de adiposidade central & 180 & $15,8(13,8-18,0)$ \\
\hline Excesso de adiposidade periférica & 129 & $11,3(9,6-13,3)$ \\
\hline Dislipidemia & 697 & $62,1(59,2-64,9)$ \\
\hline Hiperglicemia & 74 & $6,6(5,3-8,3)$ \\
\hline Pressão arterial elevada & 307 & $27,0(24,5-29,7)$ \\
\hline
\end{tabular}

* Salário mínimo de referência no período do estudo: 2011 = R\$ 545,00 e 2012 = R\$ 622,00. 
Tabela 2

Razão de prevalência (RP), intervalo de 95\% de confiança (IC95\%), análise bruta e ajustada da associação de fatores demográficos, socioeconômicos, biológicos e comportamentais com dislipidemia em escolares. Amargosa, Bahia, Brasil, 2011-2012.

\begin{tabular}{|c|c|c|c|c|c|c|}
\hline \multirow[t]{2}{*}{ Variáveis independentes } & \multicolumn{2}{|c|}{ Dislipidemia } & \multicolumn{2}{|c|}{ Análise bruta } & \multicolumn{2}{|c|}{ Análise ajustada } \\
\hline & $\mathrm{n}$ & $\%$ & RP (IC95\%) & Valor de $p$ & RP (IC95\%) & Valor de $p$ \\
\hline Sexo & & & & 0,251 * & & \\
\hline Masculino & 301 & 60,2 & 1,00 & & & \\
\hline Feminino & 396 & 63,6 & $0,95(0,86-1,04)$ & & & \\
\hline Faixa etária (anos) & & & & 0,011 * & & 0,362 * \\
\hline Criança (6-9) & 201 & 56,5 & 1,00 & & 1,00 & \\
\hline Adolescente (10-18) & 496 & 64,7 & $1,15(1,03-1,27)$ & & $1,06(0,94-1,19)$ & \\
\hline Cor da pele & & & & 0,868 * & & \\
\hline Branca & 138 & 63,0 & 1,00 & & & \\
\hline Outras & 557 & 62,0 & $0,98(0,82-1,19)$ & & & \\
\hline Localização geográfica da escola & & & & 0,001 * & & 0,001 * \\
\hline Área urbana & 577 & 67,5 & $1,51(1,31-1,74)$ & & $1,52(1,31-1,76)$ & \\
\hline Área rural & 120 & 44,8 & 1,00 & & 1,00 & \\
\hline Tipo de escola & & & & 0,278 * & & \\
\hline Pública & 636 & 61,6 & 1,00 & & & \\
\hline Particular & 61 & 67,0 & $1,09(0,93-1,27)$ & & & \\
\hline Número de irmãos & & & & 0,655 * & & \\
\hline Nenhum & 68 & 61,3 & $1,01(0,85-1,19)$ & & & \\
\hline $1-2$ & 355 & 63,5 & $1,05(0,95-1,15)$ & & & \\
\hline$\geq 3$ & 266 & 60,7 & 1,00 & & & \\
\hline Número de pessoas por residência & & & & 0,119 * & & 0,450 * \\
\hline$\leq 3$ & 152 & 63,9 & $1,12(0,98-1,29)$ & & $1,08(0,94-1,25)$ & \\
\hline $4-5$ & 361 & 63,9 & $1,12(1,00-1,26)$ & & $1,08(0,95-1,22)$ & \\
\hline$\geq 6$ & 177 & 56,9 & 1,00 & & 1,00 & \\
\hline Escolaridade materna (anos) & & & & 0,052 * & & 0,242 * \\
\hline$<4$ & 143 & 55,0 & 1,00 & & 1,00 & \\
\hline $4-8$ & 248 & 64,6 & $1,17(1,03-1,34)$ & & $1,09(0,95-1,24)$ & \\
\hline$>8$ & 262 & 63,0 & $1,15(1,00-1,31)$ & & $1,00(0,87-1,15)$ & \\
\hline Renda familiar mensal (salário mínimo) ** & & & & 0,162 * & & 0,894 * \\
\hline$<1$ & 366 & 60,5 & 1,00 & & 1,00 & \\
\hline$\geq 1$ & 293 & 64,7 & $1,07(0,97-1,17)$ & & $1,01(0,91-1,11)$ & \\
\hline Classe econômica & & & & 0,685 * & & \\
\hline$A B$ & 84 & 61,3 & 1,00 & & & \\
\hline C & 343 & 63,3 & $1,04(0,95-1,15)$ & & & \\
\hline DE & 268 & 60,6 & $1,01(0,87-1,18)$ & & & \\
\hline Índice de massa corporal & & & & 0,001 * & & 0,250 * \\
\hline Excesso de peso & 138 & 77,1 & $1,30(1,18-1,43)$ & & $1,11(0,93-1,32)$ & \\
\hline Peso normal & 559 & 59,2 & 1,00 & & 1,00 & \\
\hline Circunferência da cintura & & & & 0,001 * & & 0,001 * \\
\hline Elevada & 361 & 69,0 & $1,23(1,13-1,35)$ & & $1,20(1,10-1,32)$ & \\
\hline Normal & 336 & 56,0 & 1,00 & & 1,00 & \\
\hline Razão cintura/estatura & & & & 0,001 * & & 0,556 * \\
\hline Elevada & 167 & 75,2 & $1,28(1,17-1,40)$ & & $1,05(0,90-1,23)$ & \\
\hline Normal & 530 & 58,8 & 1,00 & & & \\
\hline Adiposidade central & & & & 0,001 * & & 0,552 * \\
\hline Elevada & 135 & 75,8 & $1,28(1,16-1,41)$ & & $1,05(0,90-1,23)$ & \\
\hline Normal & 562 & 59,5 & 1,00 & & 1,00 & \\
\hline
\end{tabular}

(continua) 
Tabela 2 (continuação)

\begin{tabular}{|c|c|c|c|c|c|c|}
\hline \multirow[t]{2}{*}{ Variáveis independentes } & \multicolumn{2}{|c|}{ Dislipidemia } & \multicolumn{2}{|c|}{ Análise bruta } & \multicolumn{2}{|c|}{ Análise ajustada } \\
\hline & $\mathbf{n}$ & $\%$ & RP (IC95\%) & Valor de $p$ & RP (IC95\%) & Valor de $p$ \\
\hline Adiposidade periférica & & & & 0,001 * & & 0,674 * \\
\hline Elevada & 97 & 75,8 & $1,26(1,13-1,40)$ & & $1,03(0,89-1,19)$ & \\
\hline Normal & 600 & 60,3 & 1,00 & & 1,00 & \\
\hline Maturação sexual & & & & $0,001 * \star \star$ & & 0,373 *** \\
\hline Pré-púbere & 275 & 56,6 & 1,00 & & 1,00 & \\
\hline Púbere & 367 & 65,7 & $1,16(1,05-1,28)$ & & $1,03(0,93-1,14)$ & \\
\hline Pós-púbere & 52 & 69,3 & $1,23(1,03-1,45)$ & & $1,08(0,91-1,27)$ & \\
\hline Prática de atividade física & & & & 0,108 * & & 0,281 * \\
\hline Insuficientemente ativo & 437 & 63,5 & $1,09(0,98-1,20)$ & & $1,06(0,96-1,17)$ & \\
\hline Ativo & 222 & 58,4 & 1,00 & & 1,00 & \\
\hline Tempo assistindo à TV (horas/dia) & & & & 0,909 * & & \\
\hline$\geq 2$ & 579 & 62,0 & $1,01(0,89-1,14)$ & & & \\
\hline$<2$ & 118 & 62,4 & 1,00 & & & \\
\hline
\end{tabular}

* Teste de Wald para heterogeneidade;

** Salário mínimo de referência no período do estudo: $2011=\mathrm{R} \$ 545,00$ e 2012 = R\$ 622,00;

*** Teste de Wald para tendência linear.

Tabela 3

Razão de prevalência (RP), intervalo de 95\% de confiança (IC95\%), análise bruta e ajustada da associação de fatores demográficos, socioeconômicos, biológicos e comportamentais com hiperglicemia em escolares. Amargosa, Bahia, Brasil, 2011-2012.

\begin{tabular}{|c|c|c|c|c|c|c|}
\hline \multirow[t]{2}{*}{ Variáveis independentes } & \multicolumn{2}{|c|}{ Hiperglicemia } & \multicolumn{2}{|c|}{ Análise bruta } & \multicolumn{2}{|c|}{ Análise ajustada } \\
\hline & $\mathrm{n}$ & $\%$ & RP (IC95\%) & Valor de $p$ & RP (IC95\%) & Valor de $p$ \\
\hline Sexo & & & & 0,239 * & & \\
\hline Masculino & 38 & 7,6 & 1,00 & & & \\
\hline Feminino & 36 & 5,8 & $0,77(0,49-1,19)$ & & & \\
\hline Faixa etária & & & & 0,531 * & & \\
\hline Criança & 21 & 5,9 & 1,00 & & & \\
\hline Adolescente & 53 & 7,0 & $1,17(0,72-1,91)$ & & & \\
\hline Cor da pele & & & & 0,303 * & & \\
\hline Branca & 18 & 8,2 & 1,00 & & & \\
\hline Outras & 55 & 6,2 & $0,76(0,44-1,29)$ & & & \\
\hline Localização geográfica da escola & & & & 0,003 * & & 0,004 * \\
\hline Área urbana & 68 & 8,0 & $3,48(1,53-7,93)$ & & $3,41(1,48-7,87)$ & \\
\hline Área rural & 6 & 2,3 & 1,00 & & 1,00 & \\
\hline Tipo de escola & & & & 0,986 * & & \\
\hline Pública & 68 & 6,6 & 1,00 & & & \\
\hline Particular & 6 & 6,6 & $0,99(0,44-2,23)$ & & & \\
\hline Número de irmãos & & & & 0,285 * & & \\
\hline Nenhum & 8 & 7,2 & $1,40(0,64-3,06)$ & & & \\
\hline $1-2$ & 43 & 7,7 & $1,49(0,91-2,45)$ & & & \\
\hline$\geq 3$ & 22 & 5,1 & 1,00 & & & \\
\hline
\end{tabular}

(continua) 
Tabela 3 (continuação)

\begin{tabular}{|c|c|c|c|c|c|c|}
\hline \multirow[t]{2}{*}{ Variáveis independentes } & \multicolumn{2}{|c|}{ Hiperglicemia } & \multicolumn{2}{|c|}{ Análise bruta } & \multicolumn{2}{|c|}{ Análise ajustada } \\
\hline & $\mathrm{n}$ & $\%$ & RP (IC95\%) & Valor de $p$ & RP (IC95\%) & Valor de $p$ \\
\hline Número de pessoas por residência & & & & 0,930 * & & \\
\hline$\leq 3$ & 16 & 6,7 & $1,07(0,56-2,04)$ & & & \\
\hline $4-5$ & 39 & 6,9 & $1,11(0,65-1,88)$ & & & \\
\hline$\geq 6$ & 19 & 6,2 & 1,00 & & & \\
\hline Escolaridade materna (anos) & & & & 0,211 * & & \\
\hline$<4$ & 11 & 4,3 & 1,00 & & & \\
\hline $4-8$ & 29 & 7,6 & $1,74(0,88-3,42)$ & & & \\
\hline$>8$ & 32 & 7,7 & $1,77(0,91-3,45)$ & & & \\
\hline Renda familiar mensal (salário mínimo) ** & & & & 0,552 * & & \\
\hline$<1$ & 37 & 6,2 & 1,00 & & & \\
\hline$\geq 1$ & 32 & 7,1 & $1,15(0,73-1,81)$ & & & \\
\hline Classe econômica & & & & 0,592 * & & \\
\hline$A B$ & 9 & 6,6 & 1,00 & & & \\
\hline C & 40 & 7,4 & $1,29(0,79-2,09)$ & & & \\
\hline $\mathrm{DE}$ & 25 & 5,7 & $1,14(0,55-2,39)$ & & & \\
\hline Índice de massa corporal & & & & 0,044 * & & 0,354 * \\
\hline Excesso de peso & 18 & 10,1 & $1,68(1,01-2,79)$ & & $0,67(0,29-1,56)$ & \\
\hline Peso normal & 56 & 6,0 & 1,00 & & 1,00 & \\
\hline Circunferência da cintura & & & & 0,944 * & & \\
\hline Elevada & 35 & 6,7 & $1,02(0,65-1,58)$ & & & \\
\hline Normal & 39 & 6,6 & 1,00 & & & \\
\hline Razão cintura/estatura & & & & 0,013 * & & 0,868 * \\
\hline Elevada & 23 & 10,4 & $1,81(1,13-2,90)$ & & $0,93(0,41-2,13)$ & \\
\hline Normal & 51 & 5,7 & 1,00 & & 1,00 & \\
\hline Adiposidade central & & & & 0,001 * & & 0,435 * \\
\hline Elevada & 22 & 12,4 & $2,23(1,39-3,57)$ & & $1,40(0,60-3,24)$ & \\
\hline Normal & 52 & 5,5 & 1,00 & & 1,00 & \\
\hline Adiposidade periférica & & & & 0,001 * & & 0,005 * \\
\hline Elevada & 21 & 16,4 & $3,06(1,91-4,89)$ & & $3,13(1,41-6,97)$ & \\
\hline Normal & 53 & 5,3 & 1,00 & & 1,00 & \\
\hline Maturação sexual & & & & 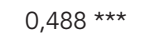 & & \\
\hline Pré-púbere & 30 & 6,3 & 1,00 & & & \\
\hline Púbere & 36 & 6,4 & $1,03(0,64-1,64)$ & & & \\
\hline Pós-púbere & 7 & 9,5 & $1,51(0,69-3,31)$ & & & \\
\hline Prática de atividade física & & & & 0,379 * & & \\
\hline Insuficientemente ativo & 49 & 7,2 & $1,25(0,77-2,03)$ & & & \\
\hline Ativo & 22 & 5,8 & 1,00 & & & \\
\hline Tempo assistindo à TV (horas/dia) & & & & 0,846 * & & \\
\hline$\geq 2$ & 62 & 6,7 & $0,94(0,52-1,71)$ & & & \\
\hline$<2$ & 12 & 6,3 & 1,00 & & & \\
\hline
\end{tabular}

* Teste de Wald para heterogeneidade;

** Salário mínimo de referência no período do estudo: $2011=\mathrm{R} \$ 545,00$ e 2012 = R\$ 622,00;

*** Teste de Wald para tendência linear. 
Tabela 4

Razão de prevalência (RP), intervalo de 95\% de confiança (IC95\%), análise bruta e ajustada da associação de fatores demográficos, socioeconômicos, biológicos e comportamentais com pressão arterial elevada em escolares. Amargosa, Bahia, Brasil, 2011-2012.

\begin{tabular}{|c|c|c|c|c|c|c|}
\hline \multirow[t]{2}{*}{ Variáveis independentes } & \multicolumn{2}{|c|}{ Pressão arterial elevada } & \multicolumn{2}{|c|}{ Análise bruta } & \multicolumn{2}{|c|}{ Análise ajustada } \\
\hline & $\mathrm{n}$ & $\%$ & RP (IC95\%) & Valor de $\mathrm{p}$ & RP (IC95\%) & Valor de $\mathrm{p}$ \\
\hline Sexo & & & & 0,129 * & & 0,404 * \\
\hline Masculino & 125 & 24,8 & 1,00 & & 1,00 & \\
\hline Feminino & 182 & 28,8 & $1,16(0,96-1,14)$ & & $1,09(0,90-1,32)$ & \\
\hline Faixa etária (anos) & & & & 0,001 * & & 0,001 * \\
\hline Criança (6-9) & 34 & 9,4 & 1,00 & & 1,00 & \\
\hline Adolescente (10-18) & 273 & 35,2 & $3,75(2,69-5,24)$ & & $2,34(1,50-3,64)$ & \\
\hline Cor da pele & & & & 0,127 * & & 0,285 * \\
\hline Branca & 49 & 22,2 & 1,00 & & 1,00 & \\
\hline Outras & 256 & 28,1 & $1,27(0,94-1,72)$ & & $1,15(0,89-1,50)$ & \\
\hline Localização geográfica da escola & & & & 0,001 * & & 0,083 * \\
\hline Área urbana & 271 & 31,4 & $2,39(1,74-3,29)$ & & $1,39(0,96-2,01)$ & \\
\hline Área rural & 36 & 13,1 & 1,00 & & 1,00 & \\
\hline Tipo de escola & & & & 0,248 * & & \\
\hline Pública & 277 & 26,6 & 1,00 & & & \\
\hline Particular & 30 & 31,9 & $1,20(0,88-1,64)$ & & & \\
\hline Número de irmãos & & & & 0,498 ** & & \\
\hline Nenhum & 28 & 25,0 & $0,89(0,62-1,27)$ & & & \\
\hline $1-2$ & 153 & 27,0 & $0,96(0,79-1,17)$ & & & \\
\hline$\geq 3$ & 125 & 28,2 & 1,00 & & & \\
\hline Número de pessoas por residência & & & & 0,222 ** & & \\
\hline$\leq 3$ & 73 & 30,5 & $1,19(0,91-1,56)$ & & & \\
\hline $4-5$ & 151 & 26,4 & $1,03(0,82-1,30)$ & & & \\
\hline$\geq 6$ & 81 & 25,6 & 1,00 & & & \\
\hline Escolaridade materna (anos) & & & & $0,800 * \star$ & & \\
\hline$<4$ & 77 & 29,3 & 1,00 & & & \\
\hline $4-8$ & 96 & 24,7 & $0,95(0,74-1,21)$ & & & \\
\hline$>8$ & 117 & 27,7 & $0,84(0,65-1,09)$ & & & \\
\hline Renda familiar mensal (salários mínimos) *** & & & & 0,062 * & & 0,676 * \\
\hline$<1$ & 154 & 25,1 & 1,00 & & 1,00 & \\
\hline$\geq 1$ & 138 & 30,2 & $1,20(0,99-1,46)$ & & $1,04(0,86-1,26)$ & \\
\hline Classe econômica & & & & 0,293 ** & & \\
\hline$A B$ & 43 & 30,7 & 1,00 & & & \\
\hline C & 149 & 27,1 & $0,88(0,66-1,17)$ & & & \\
\hline $\mathrm{DE}$ & 115 & 25,8 & $0,84(0,63-1,13)$ & & & \\
\hline Índice de massa corporal & & & & 0,001 * & & 0,273 * \\
\hline Excesso de peso & 75 & 41,4 & $1,71(1,39-2,10)$ & & $1,27(0,83-1,95)$ & \\
\hline Peso normal & 232 & 24,3 & 1,00 & & 1,00 & \\
\hline Circunferência da cintura & & & & 0,001 * & & 0,571 * \\
\hline Elevada & 175 & 33,1 & $1,52(1,26-1,85)$ & & $1,08(0,82-1,43)$ & \\
\hline Normal & 132 & 21,7 & 1,00 & & 1,00 & \\
\hline Razão cintura/Estatura & & & & 0,001 * & & 0,001 * \\
\hline Elevada & 92 & 41,1 & $1,74(1,43-2,12)$ & & $1,62(1,26-2,08)$ & \\
\hline Normal & 215 & 23,5 & 1,00 & & 1,00 & \\
\hline
\end{tabular}

(continua) 
Tabela 4 (continuação)

\begin{tabular}{|c|c|c|c|c|c|c|}
\hline \multirow[t]{2}{*}{ Variáveis independentes } & \multicolumn{2}{|c|}{ Pressão arterial elevada } & \multicolumn{2}{|c|}{ Análise bruta } & \multicolumn{2}{|c|}{ Análise ajustada } \\
\hline & $\mathrm{n}$ & $\%$ & RP (IC95\%) & Valor de $\mathrm{p}$ & RP (IC95\%) & Valor de $p$ \\
\hline Adiposidade central & & & & 0,001 * & & 0,773 * \\
\hline Elevada & 66 & 36,7 & $1,46(1,17-1,82)$ & & $1,07(0,69-1,65)$ & \\
\hline Normal & 241 & 25,2 & 1,00 & & 1,00 & \\
\hline Adiposidade periférica & & & & 0,014 * & & 0,825 * \\
\hline Elevada & 46 & 35,7 & $1,38(1,07-1,78)$ & & $0,95(0,63-1,45)$ & \\
\hline Normal & 261 & 25,9 & 1,00 & & 1,00 & \\
\hline Maturação sexual & & & & $0,001 * \star$ & & 0,007 ** \\
\hline Pré-púbere & 59 & 12,0 & 1,00 & & 1,00 & \\
\hline Púbere & 215 & 37,9 & $3,16(2,43-4,11)$ & & $1,77(1,16-2,70)$ & \\
\hline Pós-púbere & 33 & 44,0 & $3,67(2,59-5,21)$ & & $2,06(1,21-3,50)$ & \\
\hline Prática de atividade física & & & & 0,001 * & & 0,034 * \\
\hline Insuficientemente ativo & 215 & 30,9 & $1,45(1,16-1,81)$ & & $1,32(1,02-1,71)$ & \\
\hline Ativo & 82 & 21,4 & 1,00 & & 1,00 & \\
\hline Tempo assistindo à TV (horas/dia) & & & & 0,648 * & & \\
\hline$\geq 2$ & 258 & 27,3 & $0,94(0,72-1,22)$ & & & \\
\hline$<2$ & 49 & 25,7 & 1,00 & & & \\
\hline
\end{tabular}

* Teste de Wald para heterogeneidade;

** Teste de Wald para tendência linear;

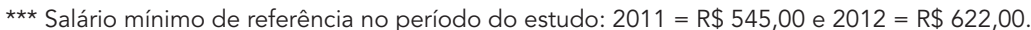

direcionamento de ações que visem à prevenção das doenças cardiovasculares no ambiente escolar ena comunidade de populações semelhantes à amostra estudada.

A elevada prevalência de dislipidemia observada no presente trabalho foi similar à reportada por outros estudos nacionais 8,9 , que utilizaram o mesmo critério de classificação para este desfecho, e superior a estudos internacionais 10,11. Em relação à hiperglicemia, nossos achados demonstraram prevalência superior à observada em escolares brasileiros 12,15 e inferior à reportada em jovens norte-americanos 14 . Para a pressão arterial elevada, a prevalência do presente estudo foi superior à descrita para as populações pediátricas brasileira 12,13,15, alemã 11 e norteamericana 14 . Este trabalho foi realizado em um município de pequeno porte e quase $60 \%$ dos investigados declararam renda familiar mensal inferior a um salário mínimo. Essas características, além de dificultar comparações devido à escassez de pesquisas que investigaram crianças e adolescentes com este perfil, sugerem que a transição epidemiológica brasileira, com elevada prevalência de fatores de risco cardiovascular, não está ocorrendo apenas em capitais e grandes centros urbanos, mas também em cidades pequenas.
As preocupantes prevalências de fatores de risco cardiovascular observadas na presente investigação precisam ser analisadas à luz dos determinantes sociais da saúde da Região Nordeste do Brasil e, consequentemente, do município estudado. Ainda que dados indiquem a diminuição das iniquidades econômicas e das condições de vida entre os segmentos sociais e as regiões brasileiras ${ }^{34}$, a população do Nordeste permanece com indicadores preocupantes de educação, ocupação, renda, habitação, saneamento básico e acesso aos serviços públicos de saúde ${ }^{34}$. Esses fatores são determinantes de desfechos cardiovasculares $35,36,37$ e podem explicar, ao menos em parte, as elevadas prevalências de dislipidemia, hiperglicemia e pressão arterial elevada da amostra investigada no presente estudo.

Fatores demográficos, socioeconômicos, biológicos e comportamentais estiveram associados aos fatores de risco cardiovascular avaliados neste estudo. Contudo, apenas os indicadores antropométricos de obesidade estiveram associados simultaneamente à dislipidemia, à hiperglicemia e à pressão arterial elevada. Esses achados corroboram dados de investigações prévias 10,11,12,14,38,39, sugerindo que a obesidade é um importante fator de risco para doenças cardiovasculares na população pediátrica. O IMC, 
por sua vez, não esteve associado a nenhum dos fatores de risco na análise ajustada. Tendo em vista que as medidas antropométricas podem apresentar multicolinearidade e que o presente trabalho tem desenho transversal, estudos de acompanhamento são necessários para analisar a utilidade deste método na triagem de fatores de risco. Não obstante, é relevante aumentar o corpo de conhecimento sobre a necessidade de incluir-se outros indicadores, como a CC, a RCEst e as DC, na rotina de avaliações em escolas, unidades de saúde da família e nas consultas pediátricas, com o intuito de rastrear e iniciar tratamento precoce de jovens com elevada quantidade de gordura corporal.

Na presente investigação, estudar em escolas urbanas aumentou a probabilidade para a presença de dislipidemia e de hiperglicemia. Além disso, houve uma tendência ( $p=0,083$ ) para que estudantes de escolas urbanas tivessem maior probabilidade para apresentar pressão arterial elevada. No entanto, a definição de grupos, rurais ou urbanos, mais expostos à presença de fatores de risco cardiovascular ainda é matéria em aberto. As informações presentes na literatura são divergentes e não conclusivas 40,41,42. Acredita-se que características específicas de países ou regiões, como diferenças étnicas, econômicas, culturais e de hábitos de vida, sejam alimentares ou de atividade física, podem mediar essa associação. No Brasil, a escassez de estudos sobre o tema dificulta comparações, indicando a necessidade de maior atenção aos contrastes demográficos (urbano $v s$. rural), nas diferentes regiões geográficas, relacionados ao processo saúde-doença de crianças e adolescentes.

Conforme descrito na literatura 38,43 , no presente estudo, adolescentes, púberes e pós-púberes apresentaram maior probabilidade de terem pressão arterial elevada. Contudo, essas associações não são sustentadas por mecanismos biológicos relacionados ao avanço da idade e do estágio maturacional durante a infância e adolescência. É esperado que a pressão arterial aumente gradualmente durante a primeira e segunda décadas de vida, da mesma forma que os pontos de corte para diagnóstico da pressão arterial elevada também são ajustados com o avanço da idade e estatura 21 . Ou seja, não deveria haver aumento da prevalência de pressão arterial elevada como consequência do simples avanço cronológico da idade. Da mesma forma, evidências indicam que a função endotelial e a elasticidade arterial são semelhantes entre jovens pré-púberes, púberes e pós-púberes 44 , indicando que elevações anormais da pressão arterial possivelmente não são decorrentes do desenvolvimento maturacional. Com base nesse contexto, acredita-se que fatores comportamentais podem mediar essas associações. Considerando estudos de acompanhamento 45,46, é possível que a maior prevalência de pressão arterial elevada entre adolescentes esteja relacionada ao maior tempo de exposição (desde a infância) a comportamentos pouco saudáveis, como hábitos alimentares inadequados e prática insuficiente de atividade física.

A prática insuficiente de atividade física também foi um fator de exposição à pressão arterial elevada na presente investigação. Entretanto, estudos que utilizaram o autorrelato para medir a atividade física são inconclusivos sobre a sua relação com a pressão arterial 47. Por outro lado, quando a avaliação da atividade física foi realizada por técnica direta, como no presente estudo, tem-se observado associações robustas e inversamente proporcionais entre essas variáveis 48 . Nesse sentido, pode ser relevante desenvolver ações de promoção da atividade física para o enfrentamento desse fator de risco cardiovascular na população investigada.

Indicadores da condição socioeconômica (como o tipo de escola, a escolaridade materna, a renda familiar e as classes econômicas) não estiveram associados aos fatores de risco cardiovascular investigados neste estudo. Embora em adultos a associação inversa de aspectos socioeconômicos com fatores de risco cardiovascular esteja bem estabelecida, especialmente em países de renda alta 49 , o corpo de evidências sobre esta associação na população pediátrica não é consensual 50, visto que algumas pesquisas relataram ausência de associação 12,42,51, outras observaram associação negativa 9,52, bem como há resultados de estudos que encontraram associação positiva 53,54 entre essas variáveis. Esses contrastes podem estar relacionados a diferenças metodológicas dos estudos, tais como, características das amostras estudadas, indicadores e instrumentos utilizados para avaliar os fatores socioeconômicos, desfechos cardiovasculares investigados e análises estatísticas utilizadas. Contudo, evidências indicam que a permanência de condições socioeconômicas desfavoráveis desde a infância está associada à maior risco cardiometabólico na vida adulta 49,55,56. Nesse sentido, o tempo de exposição a condições socioeconômicas desfavoráveis parece ser um importante fator de mediação e pode explicar, ao menos em parte, a ausência de associação entre a condição socioeconômica e fatores de risco cardiovascular durante a infância e adolescência.

A principal força da presente investigação foi a avaliação de três importantes fatores de risco para doenças cardiovasculares em uma amostra probabilística de base escolar composta por crianças e adolescentes, de ambos os sexos, 
residentes em um município de pequeno porte do interior do Nordeste do Brasil. Entretanto, fazse necessário considerar algumas limitações que podem ter repercutido na validade interna e externa do estudo. No que se refere à validade interna, o delineamento transversal da investigação não permite estabelecer relações de causa e efeito entre as variáveis de exposição e desfecho. Porém, estudos transversais representam a etapa inicial do processo de vigilância em saúde de uma comunidade e podem sugerir elementos importantes para desenhos subsequentes e medidas para mudanças. A pressão arterial foi mensurada em uma única ocasião, fato que pode ter superestimado a prevalência de pressão arterial elevada. Contudo, medidas em duas ou mais ocasiões diferentes são operacionalmente complicadas em estudos populacionais 13. A localização geográfica da escola não é sinônimo de localização de residência, ou seja, o escolar poderia, por exemplo, estudar em escola urbana e residir na área rural do município. No entanto, a escolha da escola como unidade de análise ocorreu devido ao grande potencial deste ambiente para o desenvolvimento de intervenções em saúde. Quanto à validade externa, a presente pesquisa foi realizada com uma amostra de crianças e adolescentes de um município de pequeno porte situado no interior do Estado da Bahia, com predominância de renda familiar mensal inferior a um salário mínimo, escolaridade materna inferior a oito anos de estudos e pertencente às classes econômicas C, D e E. Dessa forma, a extrapolação de nossos achados deve se limitar a grupos com perfil demográfico e socioeconômico semelhante ao estudado.
Em conclusão, as prevalências de dislipidemia, hiperglicemia e pressão arterial elevada observadas no presente estudo foram superiores às relatadas em grande parte dos achados disponíveis na literatura. Outras pesquisas são necessárias para confirmar se tais resultados são específicos da comunidade investigada ou representam um perfil cardiovascular de risco em jovens de municípios nordestinos com características semelhantes à amostra estudada. Os grupos mais expostos aos fatores de risco cardiovascular investigados foram compostos por jovens com excesso de gordura corporal, estudantes de escolas urbanas, adolescentes, púberes, pós-púberes e insuficientemente ativos. Nesse sentido, o enfrentamento da morbimortalidade por doenças cardiovasculares na vida adulta deve ser iniciado em idades precoces, com cuidado diferenciado a grupos mais expostos. A escola é um espaço institucional de convivência social que acolhe o escolar durante uma relevante parte de sua vida, com grande potencial para influenciar na adoção de modos de vida mais saudáveis. Os escolares quando bem informados podem ser multiplicadores e estimular mudanças em suas famílias, fomentando novos hábitos alimentares e de atividade física. As aulas curriculares de Educação Física nos Ensinos Fundamental e médio precisam ser incluídas nesse processo. Dessa forma, esforços são necessários para que o ambiente escolar seja mais aproveitado em políticas públicas de promoção da saúde cardiovascular durante a infância e adolescência. 


\section{Colaboradores}

T. M. B. Quadros, e A. P. Gordia participaram de todas as etapas da produção do artigo e foi responsável pela versão final. L. R. Silva, D. A. S. Silva e J. Mota colaboraram na análise dos dados, na revisão crítica do conteúdo e aprovação da versão final.

\section{Agradecimentos}

Às Secretarias Municipais de Educação e Saúde de Amargosa, Bahia, Brasil, pelo apoio para realização do estudo. O presente estudo recebeu auxílio financeiro da Fundação de Amparo à Pesquisa do Estado da Bahia (FAPESB), Coordenação de Aperfeiçoamento de Pessoal de Nível Superior (Capes), Brasil e Fundação para a Ciência e a Tecnologia (FCT), Portugal (FCT:UID/ DTP/00617/2013).

\section{Referências}

1. Barata RB. Epidemiologia social. Rev Bras Epidemiol 2005; 8:7-17.

2. Comissão Nacional sobre Determinantes Sociais da Saúde. As causas sociais das iniqüidades em saúde no Brasil. Rio de Janeiro: Editora Fiocruz; 2008.

3. Krieger N. Epidemiology and social sciences: towards a critical reengagement in the 21 st century. Epidemiol Rev 2000; 22:155-63.

4. Krieger N. A glossary for social epidemiology. J Epidemiol Community Health 2001; 5:693-700.
5. World Health Organization. Global health risks: mortality and burden of disease attributable to selected major risks. http://www.who.int/healthinfo/ global_burden_disease/GlobalHealthRisks_report full.pdf (acessado em 20/Jul/2014).

6. Bahia L, Coutinho ES, Barufaldi LA, Abreu GA, Malhao TA, Souza CP, et al. The costs of overweight and obesity-related diseases in the Brazilian public health system: cross-sectional study. BMC Public Health 2012; 12:440. 
7. Malta DC, Silva Junior JB. O Plano de Ações Estratégicas para o enfrentamento das doenças crônicas não transmissíveis no Brasil e a definição das metas globais para o enfrentamento dessas doenças até 2025: uma revisão. Epidemiol Serv Saúde 2013; 22:151-64.

8. Pereira PB, Arruda IK, Cavalcanti AM, Diniz AS. Lipid profile of schoolchildren from Recife, PE. Arq Bras Cardiol 2010; 95:606-13.

9. Nobre LN, Lamounier JA, Franceschini SC. Sociodemographic, anthropometric and dietary determinants of dyslipidemia in preschoolers. J Pediatr (Rio J.) 2013; 89:462-9.

10. Centers for Disease Control and Prevention. Prevalence of abnormal lipid levels among youths: United States, 1999-2006. MMWR Morb Mortal Wkly Rep 2010; 59:29-33.

11. Haas GM, Bertsch T, Schwandt P. Prehypertension and cardiovascular risk factors in children and adolescents participating in the community-based prevention education program family heart study. Int J Prev Med 2014; 5:50-6.

12. Ribas SA, Silva LC. Fatores de risco cardiovascular e fatores associados em escolares do Município de Belém, Pará, Brasil. Cad Saúde Pública 2014 30:577-86

13. Magliano ES, Guedes LG, Coutinho ES, Bloch KV. Prevalence of arterial hypertension among Brazilian adolescents: systematic review and meta-analysis. BMC Public Health 2013; 13:833.

14. May AL, Kuklina EV, Yoon PW. Prevalence of cardiovascular disease risk factors among US adolescents, 1999-2008. Pediatrics 2012; 129:1035-41.

15. Reuter CP, Burgos LT, Camargo MD, Possuelo LG, Reckziegel MB, Reuter EM, et al. Prevalence of obesity and cardiovascular risk among children and adolescents in the municipality of Santa Cruz do Sul, Rio Grande do Sul. São Paulo Med J 2013; 131:323-30.

16. Berenson GS. Childhood risk factors predict adult risk associated with subclinical cardiovascular disease. The Bogalusa Heart Study. Am J Cardiol 2002; 90:3L-7L.

17. Luiz RR, Magnanini MMF. A lógica da determinação do tamanho da amostra em investigações epidemiológicas. Cad Saúde Colet (Rio J.) 2000; 8: 9-28.

18. Friedewald WT, Levy RI, Fredrickson DS. Estimation of the concentration of low-density lipoprotein cholesterol in plasma, without use of the preparative ultracentrifuge. Clin Chem 1972; 18: 499-502.

19. Back Giuliano IC, Caramelli B, Pellanda L, Duncan B, Mattos S, Fonseca FH, et al. I diretriz de prevenção da aterosclerose na infância e adolescência. Arq Bras Cardiol 2005; 85:4-36.

20. American Diabetes Association. Diagnosis and classification of diabetes mellitus. Diabetes Care 2011; 34:62-9.
21. National High Blood Pressure Education Program Working Group on High Blood Pressure in Children and Adolescents. The fourth report on the diagnosis, evaluation, and treatment of high blood pressure in children and adolescents. Pediatrics 2004; 114(2 Suppl 4th Report):555-76.

22. Associação Brasileira de Empresas de Pesquisa. Critério de Classificação Econômica Brasil 2012. http://www.abep.org/new/ (acessado em 20/Nov/ 2011).

23. Gordon CC, Chumlea WCC, Roche AF. Stature, recumbent length, and weight. In: Lohman TG, Roche AF, Martorell R, editors. Anthropometric standardization reference manual. Champaign: Human Kinetics; 1988. p. 3-8.

24. Cole TJ, Bellizzi MC, Flegal KM, Dietz WH. Establishing a standard definition for child overweight and obesity worldwide: international survey. BMJ 2000; 320:1240-3.

25. World Health Organization. Measuring obesity: classification and distribution of anthropometric data. Copenhagen: World Health Organization; 1988. (Nutr UD, EUR/ICP/NUT 125).

26. Katzmarzyk PT, Srinivasan SR, Chen W, Malina RM, Bouchard C, Berenson GS. Body mass index, waist circumference, and clustering of cardiovascular disease risk factors in a biracial sample of children and adolescents. Pediatrics 2004; 114:198-205.

27. Harrison GC, Buskirk ER, Carter JEL, Johnston FE, Lohman TG, Pollack ML, et al. Skinfold thicknesses and measurement technique. In: Lohman TG, Roche AF, Martorell R, editors. Anthropometric standardization reference manual. Champaign: Human Kinetics; 1988. p. 55-70.

28. Johnson CL, Fulwood R, Abraham S, Bryner JD. Basic data on anthropometric measurements and angular measurements of the hip and knee joints for selected age groups 1-74 years of age. Vital Health Stat 11 1981; (219):1-68.

29. Tanner JM. Growth and adolescence. Oxford: Blackwell Scientific Publication; 1962.

30. Martin RHC, Uezu R, Parra SA, Arena SS, Bojikian LP, Böhme MTS. Auto-avaliação da maturação sexual masculina por meio da utilização de desenhos e fotos. Rev Paul Educ Fís 2001; 15:212-22.

31. Duncan JS, Schofield G, Duncan EK. Step count recommendations for children based on body fat. Prev Med 2007; 44:42-4.

32. American Academy of Pediatrics. American Academy of Pediatrics: Children, adolescents, and television. Pediatrics 2001; 107:423-6.

33. Norton K, Olds T. Antropométrica. Porto Alegre: Editora Artmed; 2005.

34. Instituto Brasileiro de Geografia e Estatística. Síntese de indicadores sociais: uma análise das condições de vida da população brasileira; 2013. http:// biblioteca.ibge.gov.br/visualizacao/livros/liv66777. pdf (acessado em 24/Fev/2015).

35. Nogueira MC, Ribeiro LC, Cruz OG. Desigualdades sociais na mortalidade cardiovascular precoce em um município de médio porte no Brasil. Cad Saúde Pública 2009; 25: 2321-32. 
36. Soares GP, Brum JD, Oliveira GM, Klein CH, Souza e Silva NA. Evolução de indicadores socioeconômicos e da mortalidade cardiovascular em três estados do Brasil. Arq Bras Cardiol 2013; 100:147-56.

37. Santos JP, Paes NA. Associação entre condições de vida e vulnerabilidade com a mortalidade por doenças cardiovasculares de homens idosos do nordeste. Rev Bras Epidemiol 2014; 17:407-20.

38. Rodrigues AN, Abreu GR, Resende RS, Goncalves WL, Gouvea SA. Cardiovascular risk factor investigation: a pediatric issue. Int J Gen Med 2013; 6 : $57-66$.

39. Conceicao-Machado ME, Silva LR, Santana ML, Pinto EJ, Silva RC, Moraes LT, et al. Hypertriglyceridemic waist phenotype: association with metabolic abnormalities in adolescents. J Pediatr (Rio J.) 2013; 89:56-63.

40. Ahmadi A, Gharipour M, Nouri F, Sarrafzadegan N. Metabolic syndrome in Iranian youths: a population-based study on junior and high schools students in rural and urban areas. J Diabetes Res 2013; 2013:738485.

41. McMurray RG, Harrell JS, Bangdiwala SI, Deng S. Cardiovascular disease risk factors and obesity of rural and urban elementary school children. J Rural Health 1999; 15:365-74.

42. Ochoa-Aviles A, Andrade S, Huynh T, Verstraeten R, Lachat C, Rojas R, et al. Prevalence and socioeconomic differences of risk factors of cardiovascular disease in Ecuadorian adolescents. Pediatr Obes 2012; 7:274-83.

43. Lauer RM, Anderson AR, Beaglehole R, Burns TL. Factors related to tracking of blood pressure in children. U.S. National Center for Health Statistics Health Examination Surveys Cycles II and III. Hypertension 1984; 6:307-14.

44. Marlatt KL, Steinberger J, Dengel DR, Sinaiko A, Moran A, Chow LS, et al. Impact of pubertal development on endothelial function and arterial elasticity. J Pediatr 2013; 163:1432-6.

45. Moore LL, Singer MR, Bradlee ML, Djousse L, Proctor MH, Cupples LA, et al. Intake of fruits, vegetables, and dairy products in early childhood and subsequent blood pressure change. Epidemiology 2005; 16:4-11.

46. Gopinath B, Hardy LL, Kifley A, Baur LA, Mitchell P. Activity behaviors in schoolchildren and subsequent 5-yr change in blood pressure. Med Sci Sports Exerc 2014; 46:724-9.
47. Correa Neto VG, Palma A. Blood pressure and its association with physical activity and obesity in adolescents: a systematic review. Ciênc Saúde Coletiva 2014; 19:797-818.

48. Ekelund U, Luan J, Sherar LB, Esliger DW, Griew P, Cooper A, et al. Moderate to vigorous physical activity and sedentary time and cardiometabolic risk factors in children and adolescents. JAMA 2012; 307:704-12.

49. Clark AM, DesMeules M, Luo W, Duncan AS, Wielgosz A. Socioeconomic status and cardiovascular disease: risks and implications for care. Nat Rev Cardiol 2009; 6:712-22.

50. Slopen N, Goodman E, Koenen KC, Kubzansky LD. Socioeconomic and other social stressors and biomarkers of cardiometabolic risk in youth: a systematic review of less studied risk factors. PLoS One 2013; 8:e64418.

51. Beck CC, Lopes AS, Giuliano ICB, Borgatto AS. Fatores de risco cardiovascular em adolescentes de município do sul do Brasil: prevalência e associações com variáveis sociodemográficas. Rev Bras Epidemiol 2011; 14:36-49.

52. Goodman E, McEwen BS, Huang B, Dolan LM, Adler NE. Social inequalities in biomarkers of cardiovascular risk in adolescence. Psychosom Med 2005; 67:9-15.

53. Guimarães IC, Guimarães AC. Prevalence of cardiovascular risk factors in selected samples of schoolchildren: socioeconomic influence. Prev Cardiol 2005; 8:23-8.

54. Giuliano ICB, Coutinho MS, Freitas SF, Pires MM, Zunino JN, Ribeiro RQC. Lípides séricos em crianças e adolescentes de Florianópolis, SC - Estudo Floripa Saudável 2040. Arq Bras Cardiol 2005; 85:85-91.

55. Pollitt RA, Rose KM, Kaufman JS. Evaluating the evidence for models of life course socioeconomic factors and cardiovascular outcomes: a systematic review. BMC Public Health 2005; 5:7.

56. Non AL, Rewak M, Kawachi I, Gilman SE, Loucks EB, Appleton AA, et al. Childhood social disadvantage, cardiometabolic risk, and chronic disease in adulthood. Am J Epidemiol 2014; 180:263-71. 


\section{Abstract}

This study focused on the prevalence of dyslipidemia, high blood glucose, and high blood pressure in schoolchildren and the associations with demographic, socioeconomic, biological, and behavior factors using a cross-sectional design with 1,139 schoolchildren from six to 18 years of age in Amargosa, Bahia State, Brazil. Prevalence ratio (PR) was used as the measure of association. Prevalence rates for dyslipidemia, high blood glucose, and high blood pressure were $62.1 \%, 6.6 \%$, and $27 \%$, respectively. Dyslipidemia was associated with the school's geographic location $(P R=1.52)$ and child's waist circumference $(P R=1.20)$, and high blood glucose with the school's geographic location $(P R=$ 3.41) and child's peripheral adiposity $(P R=3.13)$. High blood pressure was associated with age bracket $(P R=$ 2.34), waist-for-height ratio ( $P R=1.62)$, sexual maturation $(P R=2.06)$, and physical activity $(P R=1.32)$. Intervention programs are needed to change life habits in schoolchildren.

Students; Cardiovascular Diseases; Risk Factors; Cross-Sectional Studies

\section{Resumen}

Este trabajo tuvo como objetivo investigar la prevalencia de dislipidemia, hiperglicemia y presión arterial elevada en escolares, así como, sus asociaciones con factores demográficos, socioeconómicos, biológicos y comportamentales. Es un estudio transversal con 1.139 escolares, de seis a 18 años de edad, del municipio de Amargosa, Bahía, Brasil. Se analizaron variables demográficas, socioeconómicas, biológicas y comportamentales. Se utilizó la razón de prevalencia (RP) como medida de asociación. Las prevalencias de dislipidemia, hiperglicemia y presión arterial elevada fueron, respectivamente, $62,1 \%, 6,6 \%$ e $27 \%$. La dislipidemia se asoció con la localización geográfica de la escuela $(R P=1,52)$ y circunferencia de la cintura $(R P=$ 1,20), y la hiperglicemia con la localización geográfica de la escuela $(R P=3,41)$ y adiposidad periférica $(R P=3,13)$. La presión arterial elevada se asoció con la franja de edad $(R P=2,34)$, razón de la cintura por la estatura $(R P=1,62)$, madurez sexual $(R P=2,06)$ y actividad física $(R P=1,32)$. Son necesarios programas de intervención para escolares basados en cambios de hábitos de vida.

Estudiantes; Enfermedades Cardiovasculares; Factores de Riesgo; Estudios Transversales
Recebido em 08/Dez/2014

Versão final reapresentada em 17/Jun/2015

Aprovado em 26/Jun/2015 